\title{
Suppression of luminosity and mass-radius relation of highly magnetized white dwarfs
}

\author{
Abhay Gupta, ${ }^{1} *$ Banibrata Mukhopadhyay ${ }^{1} \dagger$ and Christopher A. Tout ${ }^{2} \ddagger$ \\ ${ }^{1}$ Department of Physics, Indian Institute of Science, Bangalore 560012, India \\ 2 Institute of Astronomy, The Observatories, Medingley Road, Cambridge CB3 OHA, UK
}

Accepted 2020 June 2. Received 2020 April 22; in original form 2020 February 14

\begin{abstract}
We explore the luminosity $L$ of magnetized white dwarfs and its effect on the massradius relation. We self-consistently obtain the interface between the electron degenerate gas dominated inner core and the outer ideal gas surface layer or envelope by incorporating both the components of gas throughout the model white dwarf. This is obtained by solving the set of magnetostatic equilibrium, photon diffusion and mass conservation equations in the Newtonian framework, for different sets of luminosity and magnetic field. We appropriately use magnetic opacity, instead of Kramer's opacity, wherever required. We show that the Chandrasekhar-limit is retained, even at high luminosity upto about $10^{-2} L_{\odot}$ but without magnetic field, if the temperature is set constant inside the interface. However there is an increased mass for large-radius white dwarfs, an effect of photon diffusion. Nevertheless, in the presence of strong magnetic fields, with central strength of about $10^{14} \mathrm{G}$, super-Chandrasekhar white dwarfs, with masses of about $1.9 M_{\odot}$, are obtained even when the temperature inside the interface is kept constant. Most interestingly, small-radius magnetic white dwarfs remain super-Chandrasekhar even if their luminosity decreases to as low as about $10^{-20} L_{\odot}$. However, their large-radius counterparts in the same mass-radius relation merge with Chandrasekhar's result at low $L$. Hence, we argue for the possibility of highly magnetized, low luminous super-Chandrasekhar mass white dwarfs which, owing to their faintness, can be practically hidden.
\end{abstract}

Key words: (stars:) white dwarfs - magnetic fields - stars: luminosity function, mass function - equation of state - radiative transfer

\section{INTRODUCTION}

Observations of several overluminous type Ia supernovae (see, Howell et al. 2006; Scalzo et al. 2010; Silverman et al. 2011) imply the existence of super-Chandrasekhar white dwarfs. It was shown earlier (Das \& Mukhopadhyay 2012, 2013; Subramanian \& Mukhopadhyay 2015) that white dwarfs with highly super-Chandrasekhar mass, $M>$ $M_{\mathrm{Ch}}=1.44 M_{\odot}$, are possible when rotation and magnetic fields are taken into consideration. It was also shown (Das \& Mukhopadhyay 2012, 2013) that when the magnetic field is greater than a critical strength of $4.414 \times 10^{13} \mathrm{G}$, such that the Larmor radius is same order of or smaller than the Compton wavelength of the electrons, the effect of Landau quantization becomes important and thus modifies the equation of state $(\mathrm{EoS})$ of the electron degenerate

*gabhay@iisc.ac.in

†bm@iisc.ac.in

łcat@ast.cam.ac.uk matter of the white dwarf. This modified EoS further gives rise to super-Chandrasekhar white dwarfs and a new limit of $2.58 M_{\odot}$. Interestingly, Sloan Digital Sky Survey (SDSS) data (Vanlandingham et al. 2005) indicate that magnetic samples of white dwarfs span the same temperature range as non-magnetic white dwarfs and support the claim that magnetic white dwarfs tend to have larger masses compared to their non-magnetic counterparts.

The existence of magnetic white dwarfs (hereinafter B-WDs) does not just explain super-Chandrasekhar mass progenitors of overluminous type Ia supernovae but also (Mukhopadhyay \& Rao 2016) B-WDs correspond to soft gamma-ray repeaters (SGRs) and anomalous X-ray pulsars (AXPs) with much lower magnetic fields than magnetars. Further, the glitch and anti-glitch of the source $1 \mathrm{E}$ $2259+586$ may be explained based on the stiffness of the magnetic field profile, mass loss and rotational energy extraction rates of a B-WD. Moreover, the peculiar radio transient of GCRT J1745-3009 can be interpreted as a white dwarf pulsar (WDP) based on the B-WD model, because its 
larger magnetic field and smaller radius could prove it is of low luminosity and further away and hence dimmer and able to evade detection.

However, the questions remain, why have not enough B-WDs yet been seen directly and why have only about a dozen overluminous type Ia supernovae implying superChandrasekhar white dwarfs have been seen so far? While Das et al. (2013) attempted to address these questions by arguing that they are rare objects as overluminous type Ia supernovae, Bhattacharya et al. (2018) explored their luminosity with the model described by Shapiro \& Teukolsky (1983) for nonmagnetic white dwarfs. Based on the photon diffusion equation and the magnetostatic balance condition, and assuming that interface parameters remain unaffected between B-WDs and nonmagnetic white dwarfs, Bhattacharya et al. (2018) found that, for a given age, the luminosity of BWDs decreases significantly, from about $10^{-6}$ to $10^{-9} L_{\odot}$ when the magnetic field strength increases from about $10^{9}$ to $10^{12} \mathrm{G}$ at the interface and hence the envelope. In fact, Valyavin et al. (2014), based on optical observation, showed that the strong magnetic field suppresses convection over the entire surface in magnetic white dwarfs, which decreases luminosity and increases cooling time scale significantly. They analysed about eight years observed data and obtained brightness and magnetic field of WD 1953-011 which is a cool white dwarf. Their results argue that the magnetic fields are expected to be common in cool, hence low luminous, white dwarfs and B-WDs are actually too young and low luminous. Indeed, in the theoretical ground, Bhattacharya et al. (2018) found the cooling rates corresponding to luminosities from about $10^{-6}$ to $10^{-9} L_{\odot}$. In this way, they studied the effect of luminosity on the outer surface layer of the white dwarf, where only ideal gas pressure exists. Because the interface between the electron degenerate core and the surface layer or envelope is very close to the surface of the white dwarf, the mass at the interface was chosen to be the total mass of the corresponding white dwarf. Thus, the characteristics of the white dwarf were obtained by solving the two differential equations of magnetohydrostatic equilibrium and photon diffusion. While this was a very good start, the choice of the same interface parameters for B-WDs and nonmagnetic white dwarfs is a big assumption. Moreover, the results based on modelling only the envelope with preassigned mass and radius should also be taken with caution, because B-WD masses and luminosities are yet be established.

Here we remove all the above assumptions and find the mass-radius relation along with luminosity of B-WDs self-consistently. Earlier, the massradius relation was rigorously explored in Newtonian (Das \& Mukhopadhyay 2012; Das et al. 2013) as well as general relativistic (Das \& Mukhopadhyay 2014; Subramanian \& Mukhopadhyay 2015; Das \& Mukhopadhyay 2015; Mukhopadhyay et al. 2017) formalisms in various magnetic field and rotational configurations, by our group. Some of these works considered poloidally as well as toroidally dominated magnetic field geometries and obtained similar results showing existence of super-Chandrasekhar white dwarfs (also see, e.g., Boshkayev et al. 2013; Franzon \& Schramm 2015; Carvalho et al. 2018). Recently Otoniel et al. (2019) revisited the problem again rigorously in general relativistic frameworks with dipole fields, but more importantly con- sidering possible instability of the matter with respect to pycnonuclear and electron capture reactions. Such stability criteria, particularly of pycnonuclear reactions, were not considered by our group in order to establish mass-radius relation mentioned above. However, still Otoniel et al. (2019) found the maximum mass of nonrotating B-WDs to be $2.14 M_{\odot}$ with central magnetic field $\sim 3.85 \times 10^{14}$ $\mathrm{G}$ with limiting central density $9.35 \times 10^{9} \mathrm{~g} \mathrm{~cm}^{-3}$, when rotation certainly would increase the mass further. According to them, above this density, pycnonuclear fusion reactions destabilize the star. This work further establishes strongly the idea of the existence of significantly superChandrasekhar white dwarf with a new mass-limit, what we initiated in 2012 with a simpler model and evolved over the years with the introduction of rigorous physics. Nevertheless, all the work neglected any possible variation of temperature in the star and its effect in the mass-radius relations. However, this is very important when luminosity of B-WDs is also planned to be explored and the aim is to understand why super-Chandrasekhar white dwarfs are hardly visible. Bhattacharya et al. (2018) included temperature gradient in assessing luminosity of B-WDs, but they did not explore mass-radius relation and confined their physics in the envelope strictly. Theirs was just one step forward compared to white dwarf luminosity investigated by Shapiro \& Teukolsky (1983) without magnetic fields. In the present work we have removed such limitations.

We model the star throughout, from the core to surface, with the finite temperature gradient in envelope determining the underlying luminosity with total pressure as the sum of the electron degenerate, ideal gas and magnetic pressures. Naturally the correct modelling leads to the dominance of electron degenerate pressure over ideal gas pressure in core and otherwise in the envelope. The radius where the ideal gas pressure dominates the degenerate pressure is defined to be the interface. We begin to solve the three differential equations for magnetostatic equilibrium, mass conservation and photon diffusion. As we mainly venture (strongly) magnetized white dwarfs, we neglect any possible convection as strong field suppresses convection effects (e.g. Canuto \& Mazzitelli 1992; Solanki 2003). Moreover, we use magnetic opacity instead of Kramer's opacity, wherever necessary.

In the next section we give an overview of the procedure to self-consistently obtain the interface of the B-WD. In section 3 , we describe the mass-radius relation including the effects of luminosity and, hence, finite temperature gradient, and magnetic fields. Subsequently, in section 4 we attempt to reinterpret the results based on total energy conservation. In section 5 we summarize the results and in section 6 conclude with some discussion.

\section{WHITE DWARF AND ITS INTERFACE}

We describe the conditions used and equations solved to formulate a method to self-consistently obtain the structure of B-WDs and the interface between the inner electron degenerate core and outer ideal gas envelope. For the present purpose, we neglect any general relativistic effect as it is not expected to influence luminosity, which mostly is the envelope phenomena. Moreover, as the main plan is to tar- 
get significantly super-Chandrasekhar white dwarfs, possible slight change in mass using general relativistic framework compared to their Newtonian counterpart does not matter. Therefore, the model equations describing magnetostatic equilibrium, photon diffusion and mass conservation, approximating stars to be spherical without losing any physics for the present purpose, respectively are

$\frac{\mathrm{d}}{\mathrm{d} r}\left(P_{\text {degenerate }}+P_{B}+P_{\text {ideal }}\right)=-\frac{G m(r)}{r^{2}}\left(\rho+\rho_{B}\right)$,

$\frac{\mathrm{d} T}{\mathrm{~d} r}=-\frac{3}{4 a c} \frac{\kappa\left(\rho+\rho_{B}\right)}{T^{3}} \frac{L_{r}}{4 \pi r^{2}}$,

$\frac{d m}{d r}=4 \pi r^{2}\left(\rho+\rho_{B}\right)$.

In these equations, $P_{\text {degenerate }}$ and $P_{\text {ideal }}$ are the electron degeneracy pressure established by Chandrasekhar (1935) and the ideal gas pressure $\left(\rho k T / \mu m_{\mathrm{p}}\right.$, where $\rho$ is the density of the matter, $k$ Boltzmann's constant, $T$ the temperature, $\mu \approx 2$ the mean molecular weight and $m_{\mathrm{p}}$ the mass of proton) respectively, $m$ is the mass enclosed at radius $r, G$ the Newton's Gravitation constant, $a$ the radiation constant, $c$ the speed of light, $L_{r}$ the luminosity at radius $r, \kappa$ the opacity, $P_{B}$ and $\rho_{B}$ the contributions from magnetic field to the pressure and density respectively and $r$ is the distance from the centre of star. As we restrict central magnetic field to $\sim 10^{14} \mathrm{G}$, the assumption of spherical star is approximately justified (Subramanian \& Mukhopadhyay 2015).

It is well-known that compact stars with strong magnetic fields (e.g. magnetars, B-WDs) tend to have additional pressure and density contributions owing to the magnetic field which can be denoted by $P_{B}=B^{2} / 8 \pi$ where field $B=\sqrt{\boldsymbol{B} \cdot \boldsymbol{B}}$ and $\rho_{B}=B^{2} / 8 \pi c^{2}$ (Sinha et al. 2013; Das \& Mukhopadhyay 2014; Bhattacharya et al. 2018). The opacity for a non-magnetized white dwarf is approximated by Kramers' formula, $\kappa=\kappa_{0} \rho T^{-3.5}$, where $\kappa_{0}=4.34 \times$ $10^{24} Z(1+X) \mathrm{cm}^{2} \mathrm{~g}^{-1}$ and $X$ and $Z$ are the mass fractions of hydrogen and heavy elements (elements other than hydrogen and helium) in the stellar interior. For a typical white dwarf, $X=0$, and we assume for simplicity the mass fraction of helium $Y=0.9$ and $Z=0.1$. The opacity is due to the bound-free and free-free transitions of electrons (Shapiro \& Teukolsky 1983). In the presence of $B$, the variation of radiative opacity with $B$ can be modelled similarly to neutron stars as $\kappa=\kappa_{B} \approx 5.5 \times 10^{31} \rho T^{-1.5} B^{-2} \mathrm{~cm}^{2} \mathrm{~g}^{-1}$ (Ventura \& Potekhin 2001), with the condition that radiative opacity should be dominant and $B / 10^{12} \mathrm{G} \geq T / 10^{6} \mathrm{~K}$, otherwise Kramers' formula should be used. For the profile of magnetic field inside the B-WD we use the profile used earlier extensively for magnetized neutron stars and B-WDs (Bandyopadhyay et al. 1997; Das \& Mukhopadhyay 2014) given by

$B\left(\frac{\rho}{\rho_{0}}\right)=B_{\mathrm{s}}+B_{0}\left[1-\exp \left(-\eta\left(\frac{\rho}{\rho_{0}}\right)^{\gamma}\right)\right]$,

where $\rho_{0}$ is a measure of density (chosen roughly 10 per cent of the central matter density of the corresponding white dwarf), $B_{\mathrm{s}}$ is the surface magnetic field, $B_{0}$ is a parameter with dimension of magnetic field and $\eta$ and $\gamma$ are dimensionless parameters which determine how exactly the field decays from the centre to surface. This can be parameterized (Das \& Mukhopadhyay 2014) with suitable constraints on pressure. Note that, as $\rho \rightarrow 0$ close to the surface of the white dwarf, $B \rightarrow B_{\mathrm{s}}$. For our work, the parameters are selected to be $\rho_{0}=10^{9} \mathrm{~g} \mathrm{~cm}^{-3}, \eta=0.8$ and $\gamma=0.9$ following Bhattacharya et al. (2018). Also, $B_{\mathrm{s}}$ and $B_{0}$ are chosen based on previous work and observations (Das \& Mukhopadhyay 2014; Bhattacharya et al. 2018).

In order to obtain a solution, luminosity is taken to be constant for simplicity and because there is no hydrogen burning or nuclear fusion taking place to power the white dwarf, so that $L_{r}=L$. Note that in principle convection may transfer a significant fraction of the total energy flux depending on magnetic field strength. It is however also known (e.g. Canuto \& Mazzitelli 1992; Solanki 2003) that strong field significantly inhibits convection. As the present venture is mostly to analyse strong field effects in white dwarfs, when even the weakest chosen surface field is no less than $10^{7} \mathrm{G}$, we can safely ignore convection from the model equations. Koester \& Chanmugam (1990) argued that, if the luminosity is considered to be constant, it is preferred to integrate the differential equations from the surface towards the centre. The surface is defined by the corresponding radius of the white dwarf. In order to solve for the set of differential equations, here by a Runge-Kutta method, the different parameters such as temperature, mass and density at the surface must be supplied. The surface temperature is obtained from the Stefan-Boltzmann law, $T_{\mathrm{s}}=\left(L / 4 \pi R^{2} \sigma\right)^{1 / 4}$, for different luminosities $L$ and the corresponding radii $R$, when $\sigma$ is the Stefan-Boltzmann constant. The surface density, which has to be several orders of magnitude smaller than the central density, is chosen such that the equations can be solved simultaneously within the tolerance limits, because both $1 \mathrm{~g} \mathrm{~cm}^{-3}$ and $10^{-10} \mathrm{~g} \mathrm{~cm}^{-3}$ can be considered to be quite small compared to $10^{4}$ to $10^{9} \mathrm{~g} \mathrm{~cm}^{-3}$ (the range of central densities for white dwarfs). The optimum range comes out to be $10^{-6}$ to $10^{-9} \mathrm{~g} \mathrm{~cm}^{-3}$ and we choose $10^{-7} \mathrm{~g} \mathrm{~cm}^{-3}$ as the surface density for all the cases. We have checked that the results are unaffected by any minor change in the surface density within the above range. Further, the mass is obtained with a shooting method by iteratively solving the equation set to satisfy the condition that the mass obtained close to the centre, at $10 \mathrm{~km}$ from the centre, integrating from surface is same as that obtained by solving mass conservation equation (3) given the solution for density profile. In this way we obtain the mass for a given radius of a white dwarf.

Now we can self-consistently obtain the interface, which is defined to be the radius at which the ideal gas pressure starts to dominate over the degenerate pressure. An example has been discussed in the Appendix to further clarify how the interface of a white dwarf is obtained in our method. It is known that the interior of a white dwarf consists completely of degenerate gas. The electrons have a large mean free path because of the filled Fermi sea, so there is a high thermal conductivity and hence a uniform temperature (Shapiro \& Teukolsky 1983). Thus, another condition can be imposed that the R.H.S. of photon-diffusion equation (2) is zero from the interface to centre and we study its effects on the mass-radius solution. We obtain interesting results on implementing this condition in non-magnetized white dwarfs. Hence, there are two possible solution cases, 


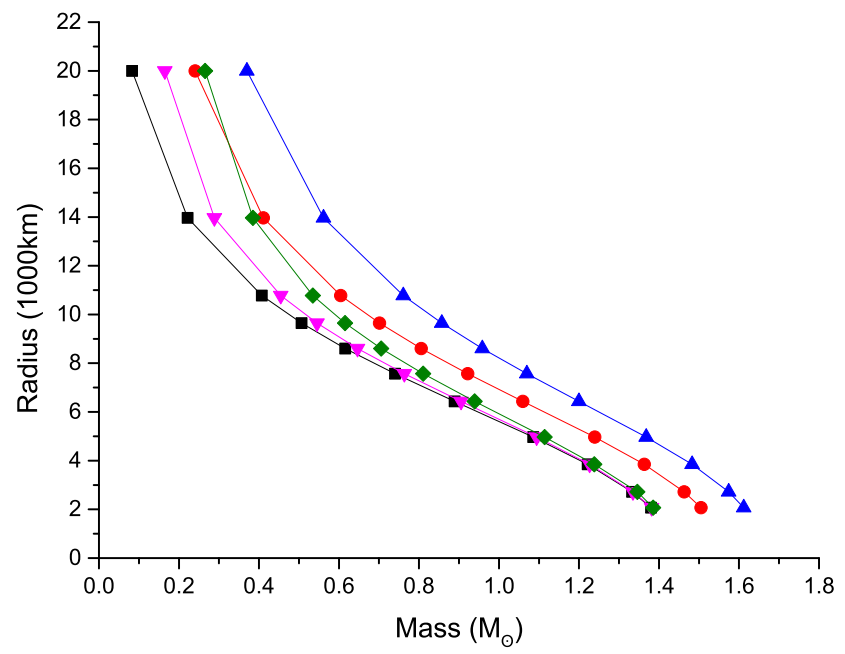

Figure 1. The effect of radiative energy transport to the massradius relation, for constant temperature throughout, Chandrasekhar's result (black squares), non-zero $\frac{d T}{d r}$ throughout for $L=10^{-2} L_{\odot}$ (blue upward triangles) and $10^{-4} L_{\odot}$ (red circles), and $\frac{d T}{d r}=0$ below the interface radius for $10^{-2} L_{\odot}$ (green diamonds) and $10^{-4} L_{\odot}$ (magenta downward triangles).

(1) the photon-diffusion equation (2) is valid throughout the white dwarf, and (2) $d T / d r=0$ for radii less than the interface radius. However, both cases produce a very similar interface radius.

\subsection{Nonmagnetic results}

First we investigate the effect of a finite temperature gradient in the stellar structure without magnetic fields. Fig. 1 shows how the photon diffusion equation, the temperature gradient, modifies the mass-radius relation compared to Chandrasekhar's original results (Chandrasekhar 1935), when $d T / d r \neq 0$ throughout, the first condition above. We find that there is a shift in the mass-radius relation towards increasing mass and radius compared to the case with $d T / d r=0$ throughout. It shows super-Chandrasekhar masses for smaller radii. However, there seems to be enough evidence that, in the absence of magnetic fields and rotation, the Chandrasekhar-limit is preserved. By imposing the second condition above, we see that indeed the Chandrasekhar limit is retained but there is an overall increase in mass in the larger radius regime in the mass-radius relation compared to Chandrasekhar's. This is similar to the earlier finding of increasing mass seen at larger radii, while preserving Chandrasekhar's limit, at higher temperatures with the finite temperature electron degenerate EoS (see fig 4. of Boshkayev et al. 2016) and the same is confirmed by SDSS. Therefore, the first condition above seems physically implausible. Note that in either of the cases, increasing luminosity leads to more deviation of the mass-radius relation with increasing mass (and radius for the first case). This is expected because higher luminosity indicates higher thermal energy leading to higher ideal gas pressure, allowing the white dwarf to hold more mass.

Table 1 lists the central and interface temperatures and densities for some white dwarf solutions of various masses and radii. It can be inferred that, for a particular radius of

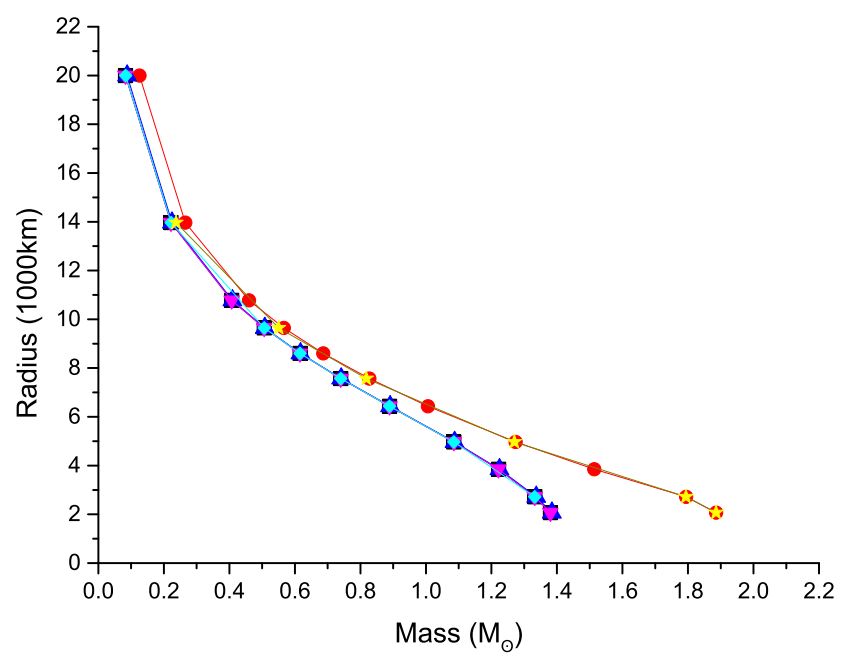

Figure 2. The effect of magnetic field to the mass-radius relation, for zero field, Chandrasekhar's results (black squares), $\left(B_{\mathrm{S}}, B_{0}\right)=$ $\left(10^{9}, 10^{14}\right) \mathrm{G}$ (red circles), $\left(10^{9}, 10^{13}\right) \mathrm{G}$ (blue upwards triangles), $\left(10^{9}, 10^{12}\right) \mathrm{G}$ (sky diamonds), $\left(10^{7}, 10^{14}\right) \mathrm{G}$ (yellow stars) and $\left(10^{7}, 10^{12}\right) \mathrm{G}$ (magenta downward triangles).

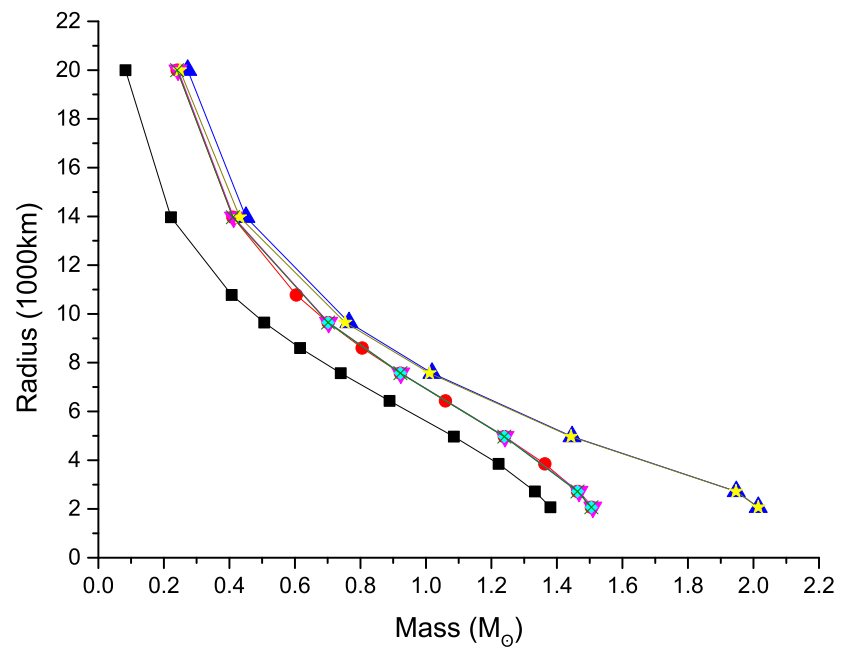

Figure 3. The effect of magnetic field and luminosity on the mass-radius relation, for Chandrasekhar's results, without thermal and field contributions (black squares), and $d T / d r \neq 0$ throughout for $\left(B_{s}, B_{0}\right)=(0,0) \mathrm{G}$ (red circles), $\left(10^{9}, 10^{14}\right) \mathrm{G}$ (blue upward triangles), $\left(10^{9}, 10^{13}\right) \mathrm{G}$ (magenta downward triangles), $\left(10^{9}, 10^{12}\right) \mathrm{G}$ (sky diamonds), $\left(10^{7}, 10^{14}\right) \mathrm{G}$ (yellow stars) and $\left(10^{7}, 10^{12}\right) \mathrm{G}$ (green cross). Except for Chandrasekhar's results, the luminosity is chosen to be $10^{-4} L \odot$.

the white dwarf, increasing the luminosity increases the effective thermal energy or temperature, leading to a larger size of the surface layer or envelope. This in turn decreases the interface radius in order to hold the excess thermal energy. Note that including the radiative transport of energy increases the central density and so also the mass, compared to Chandrasekhar's results (Chandrasekhar 1935). However, for smaller radii, when we make the temperature constant below the interface radius, condition two above, the increase in central density is not significant so that the 


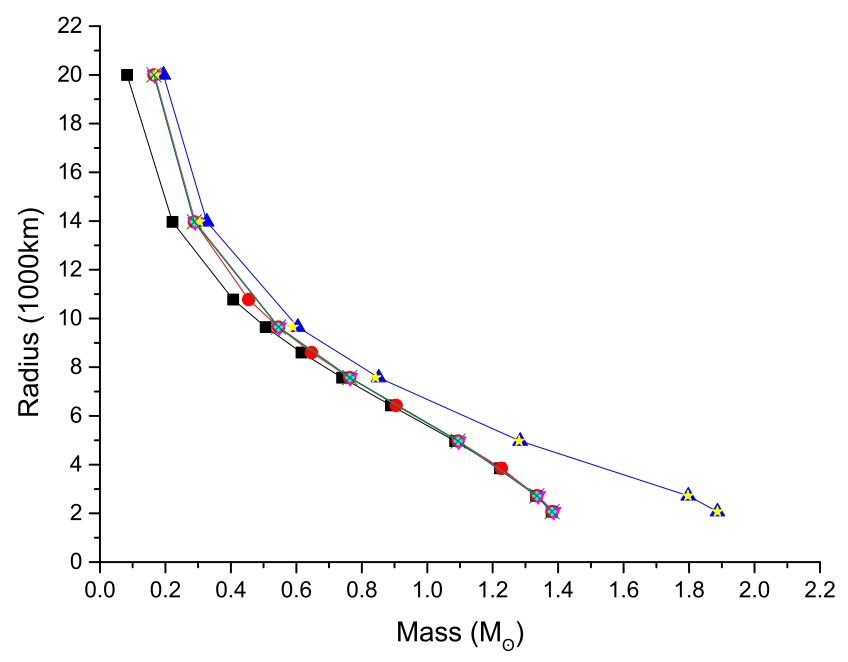

Figure 4. The effect of magnetic field and luminosity on the mass-radius relation, for Chandrasekhar's results, without thermal and field contributions (black squares), and for $d T / d r=0$ from the interface to the centre with $\left(B_{s}, B_{0}\right)=$ $(0,0) \mathrm{G}$ (red circles), $\left(10^{9}, 10^{14}\right) \mathrm{G}$ (blue upward triangles), $\left(10^{9}, 10^{13}\right) \mathrm{G}$ (magenta downward triangles), $\left(10^{9}, 10^{12}\right) \mathrm{G}$ (sky diamonds), $\left(10^{7}, 10^{14}\right) \mathrm{G}$ (yellow stars) and $\left(10^{7}, 10^{12}\right) \mathrm{G}$ (green cross). Except for Chandrasekhar's results, the luminosity is chosen to be $10^{-4} L_{\odot}$.

Chandrasekhar-limit is preserved. This is also because the surface layer is smaller for smaller white dwarfs because they are more dominated by electron degenerate matter.

\section{EFFECT OF MAGNETIC FIELD}

In order to understand how magnetic field affects white dwarf structure, we first investigate its effect on the magnetostatic equilibrium equation (1) and mass conservation equation (3) with $P_{\text {ideal }}=0$, without the photon diffusion equation (2). It is seen from Fig. 2 that $B_{0}$ plays the crucial role and it has to be large enough for any significant change in the mass-radius relation. Hence, except for the cases with $\left(B_{\mathrm{s}}, B_{0}\right)=\left(10^{9}, 10^{14}\right) \mathrm{G}$ (red circles) and $\left(10^{7}, 10^{14}\right) \mathrm{G}$ (yellow stars), all the results practically overlap.

Now if we include the photon-diffusion equation (2) in the computation, Figs 3 and 4 show how the magnetic field, along with luminosity, affects the mass-radius relation for conditions one and two above. Only strong central magnetic fields significantly affect the mass of a white dwarf for a given radius. The mass-radius relations with central field $\sim 10^{14}$ $\mathrm{G}$ merge at high mass (central density) regime exceeding Chandrasekhar's limit, as shown by the lines with blue upward triangles and yellow stars in Figs 3 and 4. Moreover, all other mass-radius relations of lower central fields also overlap with each other independent of magnetic fields, particularly in the high mass (central density) regime, restricted by Chandrasekhar's limit. However, the mass-radius curve with $d T / d r=0$ throughout (without contribution of photon diffusion) and without magnetic field, i.e. Chandrasekhar's result, shown by the line with black squares is uniquely separated from other curves, as shown in Fig. 3. This con- firms that temperature gradient and magnetic fields both have distinct effects in white dwarfs. However, Fig. 4 shows that photon diffusion with isothermal core $(d T / d r=0$ below interface), with lower central magnetic fields $<10^{14} \mathrm{G}$, only affects the mass-radius relation at the low mass (density) regime slightly, leading to increasing radius for a given mass (see lines with red circles, magenta downward triangles, sky diamonds and green cross). In the high mass (density) regime, all low magnetic field mass-radius curves practically overlap along with that of Chandrasekhar.

Notice, the central density of highly magnetized white dwarfs is higher than that of the non-magnetized ones in order to compensate for the additional magnetic pressure. This is because the contribution of the magnetic field to the total pressure is larger than that to total density. Interestingly, white dwarfs with a central field of about $10^{14} \mathrm{G}$ are significantly super-Chandrasekhar with masses exceeding $2 M_{\odot}$ at high central densities.

Table 2 shows how the presence of a magnetic field increases the central density of white dwarfs and so increases their mass. Intuitively, we know that the presence of magnetic field decreases the interface radius because it affects $\frac{d T}{d r}$ directly, similarly to the effect of luminosity on the system. Therefore, higher magnetic fields have larger effects. Nevertheless, it is crucial to know that the effective strength of the magnetic field is defined by both its $B_{\mathrm{s}}$ and $B_{0}$. If we decrease $B_{0}$ for a particular $B_{\mathrm{s}}$, the results become closer to the non-magnetic case. But for a high $B_{0}$, decreasing $B_{\mathrm{s}}$ also leads to a larger interface radius than the non-magnetic case, as seen in Table 2 when compared with the respective cases in Table 1.

\section{SUPPRESSION OF LUMINOSITY DUE TO MAGNETIC FIELD}

From the conservation of energy, in order to assure stability, the increase in the magnetic energy in the white dwarf has to be compensated for by a decrease in thermal energy for a given gravitational energy. This in turn decreases the measure of luminosity and, hence, the temperature. This is particularly the case in lower density higher radius white dwarfs, when the thermal, magnetic and gravitational energies are comparable, i.e., when the ideal gas and magnetic pressures are comparable and the degenerate gas pressure inside white dwarfs is not too high compared to them. From Figs 2, 3 and 4, we have noticed that strong central magnetic fields corresponding to $B_{0}=10^{14} \mathrm{G}$ show a deviation from the non-magnetized mass-radius relation. We explore these cases further and the results are listed in Table 3. We find that for larger radius lower density white dwarfs, a slight decrease in luminosity leads to the mass and central density of the magnetized white dwarf comparable to its non-magnetic counterpart. This is still in the observable range.

However, the situation differs for higher density smaller radius white dwarfs, where the thermal pressure inside turns out to be significantly sub-dominant, hence decreasing thermal energy does not affect its mass. Moreover, as the magnetic pressure contributes to magnetostatic balance with increasing mass for a radius same as the non-magnetic white dwarf, its central density increases compared to its nonmagnetic counterpart in order to satisfy mass equation. See 
Table 1. The effect of luminosity on the mass-radius relation where $\rho_{\mathrm{c}}, T_{\mathrm{c}}$ and $M^{\prime}$ are respectively central density, central temperature and mass, when $d T / d r=0$ below envelope, $R_{\star}, \rho_{\star}$ and $T_{\star}$ are interface radius, density and temperature respectively. $M$ is the mass for $d T / d r \neq 0$ throughout.

\begin{tabular}{ccccccccc}
\hline$R / 1000 \mathrm{~km}$ & $L / L_{\odot}$ & $\rho_{\mathrm{c}} / 10^{6} \mathrm{~g} \mathrm{~cm}^{-3}$ & $T_{\mathrm{c}} / 10^{6} \mathrm{~K}$ & $R_{\star} / 1000 \mathrm{~km}$ & $\rho_{\star} / 10^{6} \mathrm{~g} \mathrm{~cm}^{-3}$ & $T_{\star} / 10^{8} \mathrm{~K}$ & $M^{\prime} / M_{\odot}$ & $M / M_{\odot}$ \\
\hline \hline \multirow{2}{*}{20} & $10^{-4}$ & 0.0983 & 8.34 & 16.55 & 0.0011 & 0.083 & 0.165 & 0.24 \\
& $10^{-2}$ & 0.212 & 27.39 & 13.99 & 0.0068 & 0.274 & 0.265 & 0.37 \\
\hline \multirow{2}{*}{9.645} & $10^{-4}$ & 2.36 & 5.92 & 9.442 & 0.00068 & 0.059 & 0.545 & 0.7 \\
& $10^{-2}$ & 3.15 & 21.29 & 9.03 & 0.0047 & 0.213 & 0.615 & 0.86 \\
\hline \multirow{2}{*}{4.968} & $10^{-4}$ & 55.35 & 4.85 & 4.95 & 0.0005 & 0.0485 & 1.09 & 1.24 \\
& $10^{-2}$ & 60.29 & 17.97 & 4.89 & 0.0036 & 0.18 & 1.11 & 1.37 \\
\hline \multirow{2}{*}{2.068} & $10^{-4}$ & 1962 & 4.54 & 2.0651 & 0.00046 & 0.0454 & 1.381 & 1.5 \\
& $10^{-2}$ & 2019 & 16.89 & 2.0572 & 0.0034 & 0.169 & 1.385 & 1.61 \\
\hline
\end{tabular}

Table 2. The effect of magnetic field for a particular luminosity $\left(10^{-4} L_{\odot}\right)$ on the mass-radius relation where $\rho_{\mathrm{c}}, T_{\mathrm{c}}$ and $M^{\prime}$ are respectively central density, central temperature and mass, when $d T / d r=0$ below interface, $R_{\star}, \rho_{\star}$ and $T_{\star}$ are interface radius, density and temperature respectively. $M$ is the mass for $\frac{d T}{d r} \neq 0$ throughout.

\begin{tabular}{ccccccccc}
\hline$R / 1000 \mathrm{~km}$ & $\left(B_{\mathrm{s}}, B_{0}\right) / \mathrm{G}$ & $\rho_{\mathrm{c}} / 10^{6} \mathrm{~g} \mathrm{~cm}^{-3}$ & $T_{\mathrm{c}} / 10^{6} \mathrm{~K}$ & $R_{\star} / 1000 \mathrm{~km}$ & $\rho_{\star} / 10^{6} \mathrm{~g} \mathrm{~cm}^{-3}$ & $T_{\star} / 10^{8} \mathrm{~K}$ & $M^{\prime} / M_{\odot}$ & $M / M_{\odot}$ \\
\hline \hline \multirow{2}{*}{20} & $\left(10^{9}, 10^{14}\right)$ & 0.136 & 8.04 & 15.93 & 0.00108 & 0.0804 & 0.194 & 0.27 \\
& $\left(10^{9}, 10^{13}\right)$ & 0.102 & 8.3 & 16.46 & 0.00114 & 0.083 & 0.168 & 0.24 \\
& $\left(10^{7}, 10^{14}\right)$ & 0.1 & 8.27 & 16.64 & 0.0011 & 0.0827 & 0.171 & 0.25 \\
\hline \multirow{2}{*}{9.645} & $\left(10^{9}, 10^{14}\right)$ & 2.68 & 5.8 & 9.346 & 0.00066 & 0.058 & 0.604 & 0.76 \\
& $\left(10^{9}, 10^{13}\right)$ & 2.38 & 5.92 & 9.429 & 0.000683 & 0.059 & 0.547 & 0.70 \\
& $\left(10^{7}, 10^{14}\right)$ & 2.5 & 5.79 & 9.458 & 0.00066 & 0.0579 & 0.59 & 0.75 \\
\hline \multirow{2}{*}{4.968} & $\left(10^{9}, 10^{14}\right)$ & 59.17 & 4.7 & 4.9342 & 0.00049 & 0.047 & 1.284 & 1.44 \\
& $\left(10^{9}, 10^{13}\right)$ & 55.483 & 4.85 & 4.944 & 0.00051 & 0.0485 & 1.096 & 1.24 \\
& $\left(10^{7}, 10^{14}\right)$ & 58.26 & 4.64 & 4.949 & 0.000475 & 0.0465 & 1.28 & 1.44 \\
\hline \multirow{2}{*}{2.068} & $\left(10^{9}, 10^{14}\right)$ & 2502.5 & 4.23 & 2.0642 & 0.00043 & 0.0423 & 1.887 & 2.01 \\
& $\left(10^{9}, 10^{13}\right)$ & 1966.5 & 4.54 & 2.06486 & 0.000462 & 0.0454 & 1.39 & 1.51 \\
& $\left(10^{7}, 10^{14}\right)$ & 2490.3 & 4.16 & 2.066 & 0.000414 & 0.0416 & 1.887 & 2.01 \\
\hline
\end{tabular}

Table 3. Therefore, for smaller radius higher density white dwarfs, even if the luminosity decreases considerably $\left(10^{-12}\right.$ to $\left.10^{-20} L_{\odot}\right)$, the mass of the magnetized white dwarf remains quite high compared to its non-magnetic counterpart. This leads to an extended branch in the mass-radius relation which is absent in the non-magnetic counterpart. This is seen in Fig. 5. This implies that there can be white dwarfs with high magnetic field strengths (and high mass) but these are too faint to detect.

\section{DISCUSSION}

The SDSS data show that there are a large number of large radius and small mass white dwarfs whose masses are high compared to Chandrasekhar's mass-radius relation. Nevertheless, Boshkayev et al. (2016) showed that the finite temperature electron degenerate EoS at higher temperatures could explain these observations. We also obtain similar results for no and weak magnetic fields by appropriately introducing the photon diffusion equation (2). Moreover, our solutions self-consistently define the interface between the interior degenerate core and outer envelope with ideal gas layers.

In addition, we obtain super-Chandrasekhar nonmagnetic white dwarfs for a constant luminosity of, say, $10^{-2} L_{\odot}$ hypothesizing $d T / d r \neq 0$ throughout. This particularly shows the effects of very high temperatures, which arise because of the nonzero $d T / d r$ throughout and high surface temperature, on small radius white dwarfs. Further, in the presence of weak magnetic fields, we obtain the same mass-radius relations as for the non-magnetic cases when $d T / d r=0$ below the interface. However in cases of stronger magnetic fields, whether $d T / d r=0$ below the interface or not, we obtain super-Chandrasekhar masses. Thus our results, which depend on the photon diffusion equation, suggest the existence of super-Chandrasekhar white dwarfs for three different cases, (1) constant higher luminosity (about $\left.10^{-2} L_{\odot}\right)$ with nonzero $d T / d r$ throughout, (2) strong magnetic field with non-zero $\frac{d T}{d r}$ throughout, and (3) strong magnetic field with $\frac{d T}{d r}=0$ below the interface radius. While the two former situations may be implausible because then nonmagnetic and nonrotating white dwarfs might have superChandrasekhar mass, the last is quite plausible. These could 
Table 3. Mass and central density of white dwarf from conservation of magnetic and thermal energies for different field profiles and radii. Here $M_{\text {org }}$ and $\rho_{\mathrm{c}}^{\text {org }}$ denote the mass and central density of the white dwarf in the absence of magnetic field with $L=10^{-4} L_{\odot}$. Also, the central densities here are only for the cases With Interface.

\begin{tabular}{|c|c|c|c|c|c|c|c|c|c|}
\hline & & Without & Interface & & With & Interface & & & \\
\hline$R / 1000 \mathrm{~km}$ & $\left(B_{\mathrm{s}}, B_{0}\right) / \mathrm{G}$ & $L / L \odot$ & $M / M_{\odot}$ & $M_{\text {org }} / M_{\odot}$ & $L / L \odot$ & $M / M_{\odot}$ & $M_{\text {org }} / M_{\odot}$ & $\rho_{\mathrm{c}} / 10^{6} \mathrm{~g} \mathrm{~cm}^{-3}$ & $\rho_{\mathrm{c}}^{\text {org }} / 10^{6} \mathrm{~g} \mathrm{~cm}^{-3}$ \\
\hline 20 & $\left(10^{9}, 10^{14}\right)$ & $2 \times 10^{-5}$ & 0.244 & 0.24 & $8 \times 10^{-6}$ & 0.166 & 0.165 & 0.106 & 0.0983 \\
\hline 20 & $\left(10^{7}, 10^{14}\right)$ & $7 \times 10^{-5}$ & 0.242 & 0.24 & $7 \times 10^{-5}$ & 0.166 & 0.165 & 0.095 & 0.0983 \\
\hline 13.965 & $\left(10^{9}, 10^{14}\right)$ & $2 \times 10^{-5}$ & 0.416 & 0.411 & $10^{-6}$ & 0.287 & 0.288 & 0.3934 & 0.4095 \\
\hline 13.965 & $\left(10^{7}, 10^{14}\right)$ & $5 \times 10^{-5}$ & 0.415 & 0.411 & $3 \times 10^{-5}$ & 0.289 & 0.288 & 0.3854 & 0.4095 \\
\hline 9.645 & $\left(10^{9}, 10^{14}\right)$ & $5 \times 10^{-6}$ & 0.701 & 0.701 & $10^{-12}$ & 0.566 & 0.545 & 2.29 & 2.36 \\
\hline 9.645 & $\left(10^{7}, 10^{14}\right)$ & $10^{-5}$ & 0.702 & 0.701 & $10^{-12}$ & 0.551 & 0.545 & 2.121 & 2.36 \\
\hline 7.57 & $\left(10^{9}, 10^{14}\right)$ & $5 \times 10^{-7}$ & 0.923 & 0.922 & $10^{-12}$ & 0.829 & 0.763 & 7.873 & 7.738 \\
\hline 7.57 & $\left(10^{7}, 10^{14}\right)$ & $10^{-6}$ & 0.924 & 0.922 & $10^{-12}$ & 0.818 & 0.763 & 7.7563 & 7.738 \\
\hline 4.968 & $\left(10^{9}, 10^{14}\right)$ & $10^{-16}$ & 1.278 & 1.239 & $10^{-16}$ & 1.273 & 1.094 & 57.19 & 55.35 \\
\hline 4.968 & $\left(10^{7}, 10^{14}\right)$ & $10^{-12}$ & 1.285 & 1.239 & $10^{-12}$ & 1.270 & 1.094 & 56.35 & 55.35 \\
\hline 2.7215 & $\left(10^{9}, 10^{14}\right)$ & $10^{-12}$ & 1.807 & 1.463 & $10^{-16}$ & 1.794 & 1.335 & 720.89 & 686.96 \\
\hline 2.7215 & $\left(10^{7}, 10^{14}\right)$ & $10^{-12}$ & 1.807 & 1.463 & $10^{-12}$ & 1.793 & 1.335 & 718.62 & 686.96 \\
\hline 2.068 & $\left(10^{9}, 10^{14}\right)$ & $10^{-20}$ & 1.886 & 1.505 & $10^{-16}$ & 1.886 & 1.381 & 2473.8 & 1962 \\
\hline 2.068 & $\left(10^{7}, 10^{14}\right)$ & $10^{-20}$ & 1.886 & 1.505 & $10^{-16}$ & 1.886 & 1.381 & 2462.4 & 1962 \\
\hline
\end{tabular}

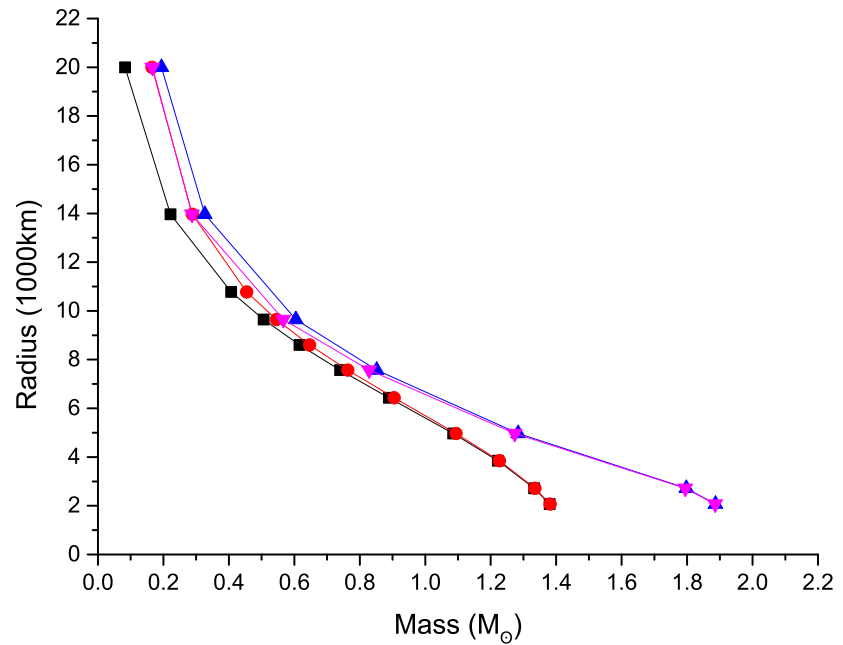

Figure 5. The effect of magnetic field optimizing luminosity to attempt to match with Chandrasekhar's mass-radius relation (black squares), for $\left(B_{\mathrm{s}}, B_{0}\right)=\left(10^{9}, 10^{14}\right) \mathrm{G}$ (magenta downward triangles), while the lines with red circles and blue upward triangles represent the results with $L=10^{-4} L_{\odot}$ for $\left(B_{\mathrm{S}}, B_{0}\right)=$ $(0,0) \mathrm{G}$ and $\left(10^{9}, 10^{14}\right) \mathrm{G}$. All cases correspond to $\frac{d T}{d r}=0$ below the interface radius. See Table 3 for specific luminosities.

very well be the candidates for super-Chandrasekhar progenitors of overluminous Type Ia supernovae.

Another interesting result is that, for the white dwarfs with smaller radii and stronger magnetic fields, the decrease in luminosity does not lead to the merging of the mass-radius relation of white dwarfs with that of their non- magnetic counterparts, leaving super-Chandrasekhar white dwarfs separate in the mass-radius relation. Hence, there can be super-Chandrasekhar magnetic white dwarfs which are so dim or have very low luminosity (as little as $\left.10^{-20} L_{\odot}\right)$, which we have missed from detection. But for larger radii, depending on their magnetic field strengths, we see the mass-radius relation merging with the nonmagnetic counterpart. Hence, with improved observational techniques and instruments, these still hold a chance to be observed because their luminosity is of the order of $10^{-5}$ to $10^{-8} L_{\odot}$.

There are a few important points to be stressed. In our case, the EoS for the electron degenerate pressure is temperature independent and it becomes very important to check for its validity when we are solving for $\frac{d T}{d r}$. In order to validate the choice, the Fermi energy $E_{F}$ has to be much greater than the thermal energy of the matter. For all the cases considered here, this condition has always been satisfied, at least in the inner degenerate core. It is observed that $E_{F} / k T \approx 5$ at the interface, and it increases below the interface.

We have attempted to explore the idea of including the energy conservation equation $\frac{d L(r)}{d r}=4 \pi r^{2} \rho(r) \epsilon(r)$, where $\epsilon(r)$ is the power produced per unit mass of stellar material in the system, in place of constant luminosity. However, because white dwarfs do not have a consistent energy production mechanism, our model equation does not perform adequately compared to our assumption of constant luminosity. Further, at high temperature, energy losses due to neutrino emission may turn out to be important (depending on the temperature and its variation), which we also have omitted from the present work. Moreover, we have not included possible convection in our computation, assuming that strong 
field inhibits its effect. However, convection may transfer significant energy (Valyavin et al. 2014) in nonmagnetic and weakly magnetized white dwarfs which we keep considering for comparison, particularly for $d T / d r \neq 0$ throughout. In future, all of these physics will be appropriately considered in a more sophisticated model framework.

In addition to this, we have used a Newtonian framework throughout our work. This is practically a valid assumption for the present purpose of white dwarfs. We eventually should explore the Tolman-Oppenheimer-Volkoff equation (Oppenheimer \& Volkoff 1939) along with temperature gradient and necessary related physics. Nonetheless, in the case of white dwarfs, $2 G M / c^{2} R$ is quite small, and this favours the choice of a Newtonian framework.

The work may be further extended for higher magnetic fields, where Landau quantization becomes important. Nonetheless, this work, in itself, is quite unique, because we could define an interface between the degenerate core and outer ideal gas envelope self-consistently and obtain massradius and luminosity relationships including the radiative transport equation in the magnetized and non-magnetized cases, which are in accordance with observations. Moreover, we have obtained a unique result that, in the presence of strong magnetic fields, small radius white dwarfs can always be undetected because they may be too dim to detect, even if they are on the similar mass-radius track as their nonmagnetic counterparts.

\section{CONCLUSION}

Observations of the light curves of over-luminous peculiar type 1a supernovae imply the presence of superChandrasekhar white dwarfs but there has been no direct observation of such a WD. Moreover, many theories have been put forward to explain the Chandrasekhar mass limit violation. These include the white dwarfs being highly magnetized and rotating. We argue that highly magnetized white dwarfs' luminosities can be suppressed to the extent that they are practically hidden, and cannot be detected. In the future, through gravitational wave detectors such as LISA, eLISA and a few others, their presence may be detected (Kalita \& Mukhopadhyay 2019).

Most of the studies of white dwarfs, so far, assumed beforehand the existence of an interface between the two pressure components, electron degenerate pressure and ideal gas pressure. We have shown that the interface can be self-consistently found, for magnetic or non-magnetic white dwarfs.

\section{ACKNOWLEDGEMENTS}

AG and BM would like to thank Mukul Bhattacharya of the University of Texas, Austin for discussion at the initial phase of the work. This work was partly supported by a project of Department of Science and Technology with Grant No.DSTO/PPH/BMP/1946 (EMR/2017/001226). CAT thanks Churchill College for his fellowship.

\section{REFERENCES}

Bandyopadhyay D., Chakrabarty S., Pal S., 1997, Physical Review Letters, 79, 2176

Bhattacharya M., Mukhopadhyay B., Mukerjee S., 2018, MNRAS, 477, 2705

Boshkayev K., Rueda J. A., Ruffini R., Siutsou I., 2013, ApJ, 762,117

Boshkayev K. A., Rueda J. A., Zhami B. A., Kalymova Z. A., Balgymbekov G. S., 2016, International Journal of Modern Physics: Conference Series, 41, 1660129

Canuto V. M., Mazzitelli I., 1992, ApJ, 389, 724

Carvalho G. A., Arbañil J. D. V., Marinho R. M., Malheiro M., 2018, European Physical Journal C, 78, 411

Chandrasekhar S., 1935, MNRAS, 95, 207

Das U., Mukhopadhyay B., 2012, Phys. Rev. D, 86, 042001

Das U., Mukhopadhyay B., 2013, Phys. Rev. Lett., 110, 071102

Das U., Mukhopadhyay $\quad$ B., 2014 , Journal of Cosmology and Astroparticle Physics, 2014, 050

Das U., Mukhopadhyay B., 2015, J. Cosmology Astropart. Phys., 2015, 016

Das U., Mukhopadhyay B., Rao A. R., 2013, ApJ, 767, L14

Franzon B., Schramm S., 2015, Phys. Rev. D, 92, 083006

Howell A. D., et al., 2006, Nature, 443, 308

Kalita S., Mukhopadhyay B., 2019, MNRAS, 490, 2692

Koester D., Chanmugam G., 1990, Reports on Progress in Physics, 53, 837

Mukhopadhyay B., Rao A. R., 2016, Journal of Cosmology and Astroparticle Physics, 2016 , 007

Mukhopadhyay B., Rao A. R., Bhatia T. S., 2017, MNRAS, 472,3564

Oppenheimer J. R., Volkoff G. M., 1939, Phys. Rev., 55, 374

Otoniel E., Franzon B., Carvalho G. A., Malheiro M., Schramm S., Weber F., 2019, The Astrophysical Journal, 879, 46

Scalzo R. A., et al., 2010, ApJ, 713, 1073

Shapiro S. L., Teukolsky S. A., 1983, Black holes, white dwarfs and neutron stars: The physics of compact objects. Wiley, New York

Silverman J. M., Ganeshalingam M., Li W., Filippenko A. V., Miller A. A., Poznanski D., 2011, MNRAS, 410, 585

Sinha M., Mukhopadhyay B., Sedrakian A., 2013, Nuclear Physics A, 898, 43

Solanki S. K., 2003, A\&ARv, 11, 153

Subramanian S., Mukhopadhyay B., 2015, MNRAS, 454, 752

Valyavin G., et al., 2014, Nature, 515, 88

Vanlandingham K. M., et al., 2005, The Astronomical Journal, 130,734

Ventura J., Potekhin A., 2001, astro-ph/0104003

\section{APPENDIX A: REPRESENTATIVE SAMPLE CASE STUDY}

Here we outline the process of obtaining the interface radius for a particular case. The following example is for $R=4968 \mathrm{~km}$ white dwarf in the presence of magnetic field $\left(B_{\mathrm{s}}, B_{0}\right)=\left(10^{9}, 10^{14}\right) \mathrm{G}$ with luminosity $10^{-4} L_{\odot}$.

In Fig. A1, we show the various pressure profiles and it is seen that roughly at $4934 \mathrm{~km}$, the ideal gas pressure overpowers the electron degeneracy pressure. Hence, this radius can be defined as the interface radius for this case. Fig. A1 clearly confirms the dominance of the electron degenerate matter in the core about three orders of magnitude over the 
ideal gas pressure. Further, applying $\frac{d T}{d r}=0$ below this interface radius, we obtain the mass for $R=4968 \mathrm{~km}$ white dwarf, see Table 2. Fig. A2 represents the profiles for density, mass, temperature and magnetic field as functions of radius. Density and magnetic field have roughly the same profiles because the strength of magnetic field is density dependent. Fig. A3 also confirms that Fermi energy is significantly dominant over thermal energy, assuring the validity of zero-temperature EoS.

This paper has been typeset from a $\mathrm{TE}_{\mathrm{E}} \mathrm{X} / \mathrm{LATE} \mathrm{X}$ file prepared by the author. 

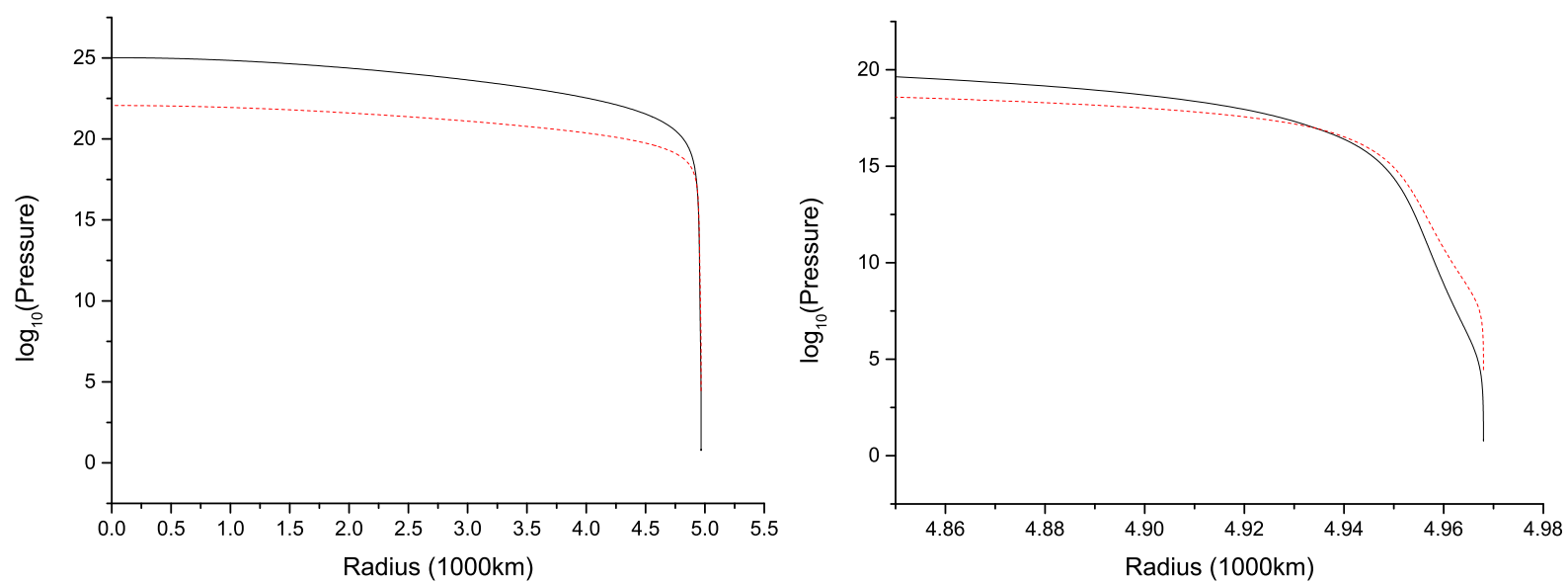

Figure A1. The variation of electron degenerate pressure (solid black line) and ideal gas pressure (dash red line) as functions of radius, when $d T / d r=0$ below interface. The right panel is the same as left panel, but zoomed in the outer region close to the surface. Note, the ideal gas pressure takes over the electron degenerate pressure around $4934 \mathrm{~km}$.
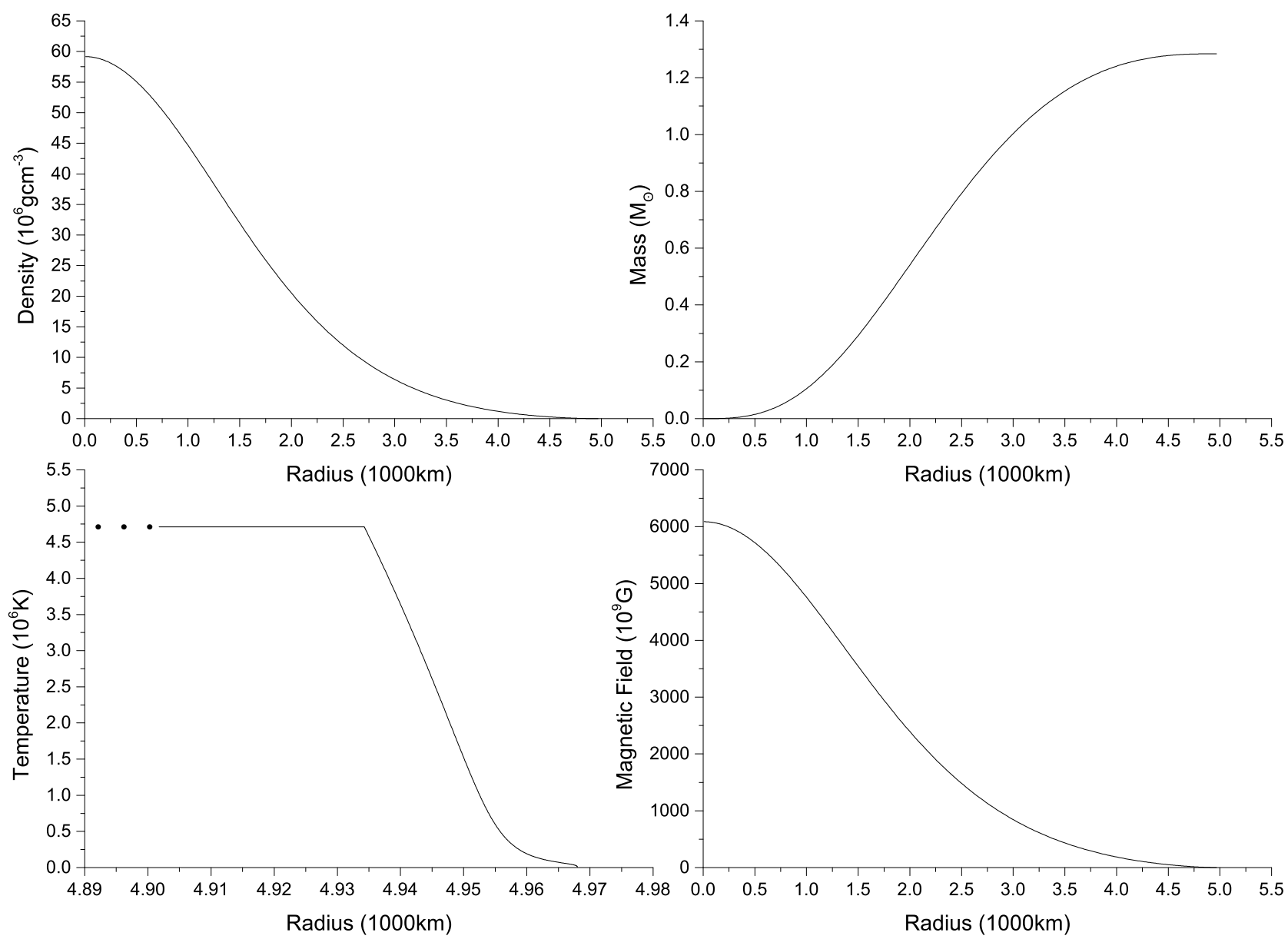

Figure A2. For a particular mass-radius, the variations of density (top left), mass (top right), temperature (bottom left) and magnetic field (bottom right), as functions of radius in a white dwarf. Note that the temperature varies only on the surface layer above $4934 \mathrm{~km}$ (interface) and remains constant from the interface to centre, part of which has been depicted. 


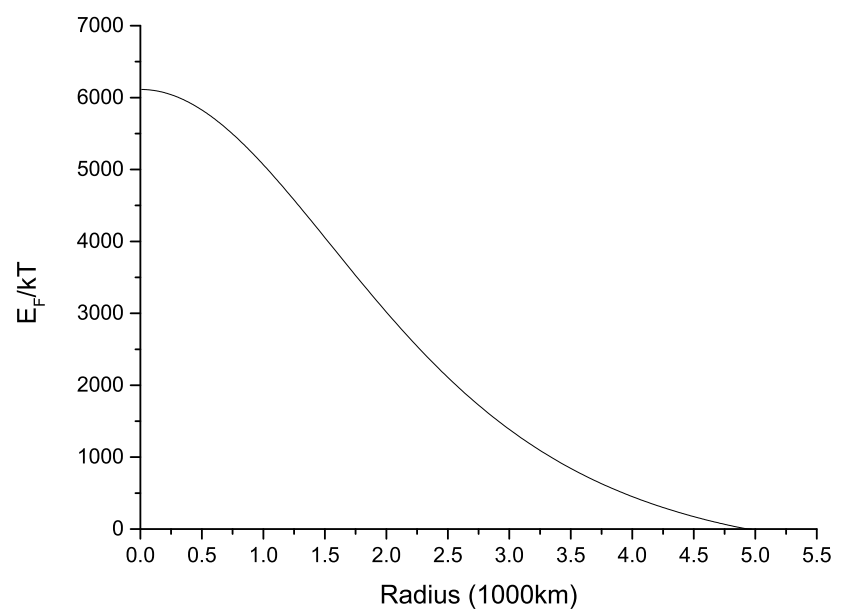

Figure A3. The variation of ratio of the Fermi energy to thermal energy as a function of radius. 